\title{
Patterns of food consumption among vegetarians and non-vegetarians
}

\author{
Michael J. Orlich ${ }^{1,2 *}$, Karen Jaceldo-Siegl ${ }^{1}$, Joan Sabaté ${ }^{1,3}$, Jing Fan ${ }^{1}$, Pramil N. Singh ${ }^{1}$ and \\ Gary E. Fraser ${ }^{1,2,3}$ \\ ${ }^{1}$ Adventist Health Studies, School of Public Health, Loma Linda University, 24951 North Circle Drive, Nichol Hall 2031, \\ Loma Linda, CA 92350, USA \\ ${ }^{2}$ Department of Preventive Medicine, School of Medicine, Loma Linda University, Loma Linda, CA USA \\ ${ }^{3}$ Department of Medicine, School of Medicine, Loma Linda University, Loma Linda, CA, USA
}

(Submitted 18 April 2014 - Final revision received 22 July 2014 - Accepted 26 July 2014 - First published online 23 September 2014 )

\begin{abstract}
Vegetarian dietary patterns have been reported to be associated with a number of favourable health outcomes in epidemiological studies, including the Adventist Health Study 2 (AHS-2). Such dietary patterns may vary and need further characterisation regarding foods consumed. The aims of the present study were to characterise and compare the food consumption patterns of several vegetarian and non-vegetarian diets. Dietary intake was measured using an FFQ among more than 89000 members of the AHS- 2 cohort. Vegetarian dietary patterns were defined a priori, based on the absence of certain animal foods in the diet. Foods were categorised into fifty-eight minor food groups comprising seventeen major food groups. The adjusted mean consumption of each food group for the vegetarian dietary patterns was compared with that for the non-vegetarian dietary pattern. Mean consumption was found to differ significantly across the dietary patterns for all food groups. Increased consumption of many plant foods including fruits, vegetables, avocados, non-fried potatoes, whole grains, legumes, soya foods, nuts and seeds was observed among vegetarians. Conversely, reduced consumption of meats, dairy products, eggs, refined grains, added fats, sweets, snack foods and non-water beverages was observed among vegetarians. Thus, although vegetarian dietary patterns in the AHS-2 have been defined based on the absence of animal foods in the diet, they differ greatly with respect to the consumption of many other food groups. These differences in food consumption patterns may be important in helping to explain the association of vegetarian diets with several important health outcomes.
\end{abstract}

Key words: Dietary patterns: Vegetarians: Foods

Given the large number of challenges faced during epidemiological investigations of diet and chronic disease relationships at the nutrient level, more attention has recently been paid to dietary patterns ${ }^{(1)}$. However, defining dietary patterns that differ in important ways and may thus have differing effects on health can be challenging. Dietary patterns have been defined using two broad approaches: data-driven approaches using pattern analysis methods and hypothesis-driven approaches using a priori definitions or scoring systems ${ }^{(2)}$.

Investigators in the Adventist Health Studies have utilised a hypothesis-driven approach to define dietary patterns according to the index of animal food avoidance. The vegetarianspectrum dietary patterns derived from this approach have been predictive of a number of important differences in health status, including obesity ${ }^{(3)}$, the metabolic syndrome ${ }^{(4)}$, diabetes mellitus type $2^{(3,5)}$, hypertension ${ }^{(6)}$ and mortality ${ }^{(7)}$. In the Adventist Health Study 2 (AHS-2), five vegetarian-spectrum dietary categories have been defined in order of increasing avoidance of animal food consumption: non-vegetarian; semivegetarian; pesco-vegetarian; lacto-ovo-vegetarian; vegan.

It is important to characterise how these dietary patterns may differ with respect to a variety of potentially important foods and nutrients. In a previous paper, Rizzo et al. ${ }^{(8)}$ had characterised these dietary patterns with respect to their nutrient profiles. In the present study, an analysis of their differences in terms of the consumption of key foods and food groups was carried out.

\section{Methods}

\section{Study population}

The AHS-2 cohort comprises more than 96000 Seventh-day Adventist men and women recruited from the USA and Canada between 2002 and $2007^{(9)}$. A detailed description of

Abbreviations: AHS-2, Adventist Health Study 2; EPIC, European Prospective Investigation into Cancer and Nutrition; NDS-R, Nutrition Data System for Research. 
the cohort characteristics and formation is given in Butler et $a l .{ }^{(9)}$. Written informed consent was obtained upon enrolment. The institutional review board of Loma Linda University approved the study.

A cross-sectional analysis was carried out in the present study, and all data used in the analysis were derived from the study baseline assessment. From the group of 96060 subjects with completely processed dietary intake data, subjects with improbable response patterns in questionnaire data (e.g. identical high-frequency responses to all questions on a page) ( $n$ 251), more than sixty-nine missing values in dietary intake data ( $n$ 2052), estimated energy intake (before imputation) greater than $18828 \mathrm{~kJ} / \mathrm{d}(4500 \mathrm{kcal} / \mathrm{d} ; n$ 2143), age missing or $<25$ years ( $n$ 262), missing value for sex ( $n$ 33), missing value for race ( $n$ 997), and estimated energy intake (after imputation) less than $2092 \mathrm{~kJ} / \mathrm{d}(500 \mathrm{kcal} / \mathrm{d})$ or greater than $18828 \mathrm{~kJ} / \mathrm{d}$ ( $4500 \mathrm{kcal} / \mathrm{d}$ ) (average $n$ 867) were excluded. After exclusion, five analytical datasets remained for use in multiple imputation analysis with an average of 89455 subjects.

\section{Measurement of dietary intake}

Methods used for measuring dietary intake in the AHS-2 have been described in detail previously ${ }^{(10,11)}$. A self-administered quantitative FFQ was used at baseline to assess usual dietary intake during the previous year. As described by Jaceldo-Siegl et $a .^{(10)}$, the FFQ was originally designed to include foods commonly consumed by US Adventists and was later revised to reduce the respondents' fatigue and to accommodate foods specific to black Adventists of US and Caribbean origin. All versions of the FFQ consist of two major sections: (1) a food list that includes 130-141 items of fruits, vegetables, legumes, grains, oils, dairy products, meats, fish, eggs, sweets, snacks, condiments, mixed foods, and beverages and (2) sixty-three to seventy-nine items of commercially prepared products, such as dietary supplements, dry cereals and vegetarian protein products that require respondents to examine food labels. For each hard-coded food item, estimates of both frequency of consumption (seven to nine categories) and serving size (three categories: standard, half or less, and one and a half or more) were elicited. Similarly, for each write-in food item, the participants were asked to provide details regarding frequency of consumption (seven to nine categories) and serving size (either three categories as above or write in the usual serving size). Frequency categories ranged from never or rarely up to two to six servings per $\mathrm{d}$ depending on food type. Portion sizes included a given standard serving (e.g. cup, tablespoon or slice), and pictures portraying serving sizes of common foods or beverages were included in the questionnaire to assist the participants in estimating portion sizes ${ }^{(11)}$.

Daily food intake estimates (in $\mathrm{g}$ or $\mathrm{kJ}$ ) were calculated using the product-sum method (see Jaceldo et al. ${ }^{(10)}$ for details). Energy and nutrient conversions were computed using the Nutrition Data System for Research (NDS-R) version 4.06 or 5.0 (Nutrition Coordinating Center) and were based on the NDS-R 2008 database. Considerable attention was paid to obtaining information on foods not found in the NDS-R database as described previously ${ }^{(10)}$.
The FFQ has been validated previously against six $24 \mathrm{~h}$ dietary recalls for intake of nutrients ${ }^{(11)}$ and selected foods and food groups ${ }^{(10)}$. On average, energy-adjusted de-attenuated validity correlations were 0.60 in whites and 0.52 in blacks across the fifty-one nutrients ${ }^{(11)}$. For foods and food groups, the average de-attenuated validity correlations were 0.59 in whites and 0.43 in blacks ${ }^{(10)}$.

\section{Definitions of dietary patterns}

As described by Orlich et $a l .{ }^{(7)}$, dietary patterns were determined according to the reported intake of foods of animal origin. Thus, vegans consumed eggs/dairy products, fish and all other meats $<1$ time/month; lacto-ovo-vegetarians consumed eggs/dairy products $\geq 1$ time/month but fish and all other meats $<1$ time/month; pesco-vegetarians consumed fish $\geq 1$ time/month but all other meats $<1$ time/month; semi-vegetarians consumed non-fish meats $\geq 1$ time/month and all meats combined (fish included) $\geq 1$ time/month but $\leq 1$ time/week; and lastly, non-vegetarians consumed nonfish meats $\geq 1$ time/month and all meats combined (fish included) $>1$ time/week.

\section{Categorisation of foods}

Similar food items were grouped with consideration given to biological distinctions (e.g. fruits), commonly accepted food categories (e.g. vegetables) and certain diet-disease hypotheses (e.g. processed meats) to create fifty-eight non-overlapping minor food groups. These were further clustered into seventeen major food groups (see online supplementary Table S1).

Whenever possible, both hard-coded and write-in food items were categorised such that they contributed to the food groups as whole foods, rather than at the ingredient level. For example, the item 'French bread' was placed in the questionnaire such that it contributed its entire weight in $g$ and energy content to the food group 'Refined grains', rather than breaking it down to its constituent ingredients (flour, oil, water, etc.) and placing these into separate food groups. This was done for the vast majority of food items. A few hard-coded food items (e.g. pizza) seemed by nature to warrant categorisation at the ingredient level. For such items, a representative recipe was developed and used to divide the item into its constituent ingredients (flour, oil, cheese, tomato, etc.), which were each then placed into appropriate food groups.

Several of the groups constitute very minor components of the diet, may not generally be thought of as 'food', or do not seem to aid in comparative characterisations of dietary patterns, and thus these groups were not included in the comparative analysis described in the 'Results' section. The following groups were omitted: water from recipes; mixed foods; condiments; yeast; salt; supplements (see online supplementary Table S1).

\section{Covariates}

Other variables (all measured at baseline) were age (in years), sex (male $v$. female), race (black $v$. non-black). The participants self-identified their race/ethnicity in one or more of the 
twenty-one categories. Those self-identifying as at least part black/African American, West Indian/Caribbean, African or other black were categorised as black for this analysis and all others as non-black.

\section{Statistical analyses}

For the entire sample, unadjusted descriptive statistics were calculated for each food group. Adjusted mean values of all food groups were calculated for each dietary pattern. Mean values were adjusted for age, sex and race by direct standardisation, using the entire analytical sample as the standard distribution. The mean values of these dietary patterns were also standardised to an $8368 \mathrm{~kJ} / \mathrm{d}(2000 \mathrm{kcal} / \mathrm{d})$ diet as follows: intake values of each food item for each participant were divided by the total daily energy intake of the participant and multiplied by 8368 to yield the standardised intake; these standardised intake values were used to compute the mean values by dietary pattern. The relative mean consumption of food groups for each vegetarian dietary pattern (compared with the non-vegetarian dietary pattern) was calculated as the ratio of the standardised mean in $g$ for each vegetarian dietary pattern to the standardised mean in $\mathrm{g}$ for the non-vegetarian dietary pattern. The $95 \% \mathrm{CI}$ for the relative mean consumption (i.e. ratio of two means) was calculated using a joint standard error estimate for the ratio of the two means, according to the following formula (a simplification of Fieller's theorem $\left.{ }^{(12)}\right): \quad \mathrm{SE}_{Q}=Q \times\left(\mathrm{SE}_{A}^{2} / A^{2}+\mathrm{SE}_{B}^{2} / B^{2}\right)^{0.5}$, where $Q$ is the ratio of the means $(Q=A / B), A$ is the first mean and $B$ is the second mean (i.e. non-vegetarian). The simplification is justified because $\mathrm{SE}_{B} \ll B$, with this being true for all dietary variables. Significance testing for differences across the dietary patterns was conducted as follows. For each food group, the null hypothesis was that none of the adjusted mean values of the four vegetarian groups differed from the adjusted mean value of the non-vegetarian group. This was tested by computing a $\chi^{2}$ test with $4 \mathrm{df}$ using the variances of each adjusted mean value. A nominal $\alpha$ value of 0.05 was selected. This process was repeated for a total of sixty-six unique significance tests (for fifty-five minor groups plus eleven major groups that were not identical to minor groups); thus a Bonferroni correction for multiple testing was applied, which yielded a corrected $\alpha$ value of 0.05/66 $=0.0008$. Multiple imputation of missing values was done for the small amount of missing data for the dietary variables used to calculate vegetarian status and food categories as we had evidence that many of the missing data were non-zero; a guided multiple imputation approach was utilised where possible ${ }^{(13,14)}$. Analyses were carried out using SAS 9.3 (SAS Institute, Inc.) and $\mathrm{R}$ version 2.13.1 (R Foundation for Statistical Computing) ${ }^{(15)}$ with the Hmisc package ${ }^{(16)}$.

\section{Results}

\section{Food consumption in the entire sample}

Unadjusted measures of the consumption of select major and minor food groups for the entire analytical sample are given in Table 1. Daily consumption for each dietary pattern is described by giving the mean value, standard deviation, 10th percentile, 25 th percentile, median, 75th percentile, 90th percentile and the percentage of responses that indicated zero intake. The mean, standard deviation and quantiles are given in units of mass $(\mathrm{g})$. The energy density of each food group is also listed to allow for approximate conversion from mass in $\mathrm{g}$ to energy content in $\mathrm{kJ}$. Mean values were consistently higher than median values, indicating a rightskewed distribution of consumption. This was much accentuated for foods that had high percentages of zero intake; in these cases, the median value was sometimes zero.

\section{Food consumption by dietary pattern: major food groups}

For each dietary pattern, the amounts of both major and minor food groups consumed (in g), adjusted for age, sex and race and standardised to an $8368 \mathrm{~kJ} / \mathrm{d}(2000 \mathrm{kcal} / \mathrm{d})$ diet, are given in Table 2. $P$ values for all major food groups were less than $0 \cdot 0001$, indicating that the vegetarian dietary patterns differed significantly (i.e. $P<$ Bonferroni-corrected $\alpha$ value of 0.0008) from the non-vegetarian dietary pattern with respect to the consumption of all major food groups.

Differences in the consumption of major food groups among the dietary patterns, expressed as the relative mean consumption of each food group for each vegetarian dietary pattern compared with the mean consumption of that food group for the non-vegetarian dietary pattern, after adjustment for age, sex and race and standardisation to an $8368 \mathrm{~kJ} / \mathrm{d}$ $(2000 \mathrm{kcal} / \mathrm{d})$ diet, are graphically illustrated in Fig. 1. As expected, given the definitions of dietary patterns, vegetarians consumed less meat, eggs and dairy products when compared with non-vegetarians. Vegans and lacto-ovovegetarians consumed negligible amounts of meats and pesco-vegetarians and semi-vegetarians consumed much less meat when compared with non-vegetarians. Vegans consumed the lowest amounts of eggs and dairy products and non-vegetarians the highest amounts, with the other vegetarian groups consuming intermediate amounts. Vegetarians also consumed lower amounts of added fats, sweets, snack foods and non-water beverages: in each case, vegans consumed the lowest amounts, non-vegetarians the highest amounts and the other vegetarian groups intermediate amounts. Vegans consumed less than one-third of the quantity of non-water beverages daily consumed by non-vegetarians and less than one-fifth of the amount of sweets. On the other hand, vegetarians consumed higher amounts of most of the other major groups of foods of plant origin when compared with non-vegetarians, including legumes, soya foods and meat analogues, nuts and seeds, grains, potatoes, avocados, fruits and vegetables. In the case of almost all the major plant food groups - legumes, soya foods and meat analogues, nuts and seeds, grains, potatoes, avocados, fruits and vegetables - vegans were found to consume the highest amounts of daily energy from these food groups, nonvegetarians the lowest amounts and the other vegetarian groups intermediate amounts. 
Table 1. Measures of daily consumption of food groups for all participants*

(Mean values and standard deviations; medians and percentiles)

\begin{tabular}{|c|c|c|c|c|c|c|c|c|c|}
\hline Foods & $\begin{array}{l}\text { Mean } \\
(\mathrm{g})\end{array}$ & $\begin{array}{l}\text { SD } \\
(g)\end{array}$ & $\begin{array}{l}\text { 10th percentile } \\
\text { (g) }\end{array}$ & $\begin{array}{c}25 \text { th } \\
\text { percentile } \\
\text { (g) }\end{array}$ & $\begin{array}{l}\text { Median } \\
\text { (g) }\end{array}$ & $\begin{array}{c}\text { 75th } \\
\text { percentile } \\
\text { (g) }\end{array}$ & $\begin{array}{l}\text { 90th } \\
\text { percentile } \\
\text { (g) }\end{array}$ & $\begin{array}{c}\text { Zero } \\
\text { intake† (\%) }\end{array}$ & $\begin{array}{c}\text { Density } \neq \\
(\mathrm{kJ} / \mathrm{g})\end{array}$ \\
\hline \multicolumn{10}{|l|}{ Major food groups } \\
\hline Fruits & $330 \cdot 2$ & $292 \cdot 6$ & $70 \cdot 7$ & $145 \cdot 6$ & $261 \cdot 8$ & $421 \cdot 2$ & $644 \cdot 9$ & 0.4 & $2 \cdot 76$ \\
\hline Vegetables & $327 \cdot 1$ & $230 \cdot 8$ & $107 \cdot 0$ & $173 \cdot 8$ & $276 \cdot 3$ & $415 \cdot 8$ & $596 \cdot 6$ & 0.0 & 1.42 \\
\hline Avocados & $7 \cdot 0$ & $14 \cdot 1$ & 0.0 & 0.0 & $4 \cdot 3$ & $6 \cdot 4$ & $27 \cdot 4$ & $42 \cdot 6$ & $5 \cdot 19$ \\
\hline Potatoes & 38.2 & $32 \cdot 8$ & $9 \cdot 0$ & $15 \cdot 1$ & $28 \cdot 9$ & $52 \cdot 6$ & $79 \cdot 0$ & 1.9 & 5.44 \\
\hline Grains & $296 \cdot 4$ & $179 \cdot 4$ & $104 \cdot 8$ & $163 \cdot 8$ & $262 \cdot 0$ & $388 \cdot 8$ & $532 \cdot 8$ & 0.1 & $7 \cdot 45$ \\
\hline Legumes & $62 \cdot 0$ & 63.0 & $10 \cdot 2$ & $23 \cdot 2$ & $43 \cdot 3$ & $80 \cdot 9$ & $134 \cdot 5$ & 3.9 & 4.98 \\
\hline $\begin{array}{l}\text { Soya foods and } \\
\text { meat analogues }\end{array}$ & $125 \cdot 2$ & $166 \cdot 0$ & $0 \cdot 0$ & $22 \cdot 6$ & $67 \cdot 8$ & $165 \cdot 0$ & $309 \cdot 1$ & $10 \cdot 7$ & $4 \cdot 39$ \\
\hline Nuts and seeds & $23 \cdot 6$ & $25 \cdot 7$ & $3 \cdot 0$ & 6.9 & $16 \cdot 1$ & 31.4 & $51 \cdot 8$ & $2 \cdot 5$ & 24.98 \\
\hline Meat & $27 \cdot 9$ & $41 \cdot 0$ & 0.0 & 0.0 & 11.4 & $41 \cdot 2$ & 81.5 & $37 \cdot 2$ & $8 \cdot 12$ \\
\hline Dairy products & $146 \cdot 3$ & $209 \cdot 6$ & 0.9 & $15 \cdot 3$ & $65 \cdot 6$ & $204 \cdot 9$ & $361 \cdot 2$ & 9.7 & $3 \cdot 39$ \\
\hline Eggs & 9.6 & $15 \cdot 9$ & 0.0 & 0.0 & $4 \cdot 1$ & $17 \cdot 5$ & $26 \cdot 2$ & $26 \cdot 1$ & $8 \cdot 41$ \\
\hline Added fats & $41 \cdot 3$ & $30 \cdot 1$ & $10 \cdot 7$ & $20 \cdot 1$ & 34.9 & $54 \cdot 8$ & $79 \cdot 4$ & 0.2 & $24 \cdot 23$ \\
\hline Sweets & 39.4 & $53 \cdot 6$ & 0.4 & $10 \cdot 0$ & $23 \cdot 0$ & $46 \cdot 8$ & $97 \cdot 4$ & $6 \cdot 8$ & 11.05 \\
\hline Snack foods & 3.6 & 5.9 & 0.0 & 0.6 & $2 \cdot 0$ & $4 \cdot 3$ & $9 \cdot 1$ & 19.5 & $26 \cdot 69$ \\
\hline Beverages & $419 \cdot 7$ & $473 \cdot 2$ & $33 \cdot 3$ & $115 \cdot 0$ & $282 \cdot 4$ & $555 \cdot 3$ & 964.0 & 3.6 & $1 \cdot 17$ \\
\hline Drinking-water & 1122.9 & $594 \cdot 6$ & $236 \cdot 9$ & $592 \cdot 3$ & $1066 \cdot 1$ & 1539.9 & $1599 \cdot 1$ & $2 \cdot 3$ & 0.00 \\
\hline \multicolumn{10}{|l|}{ Minor food groups } \\
\hline Citrus fruits & $73 \cdot 0$ & $103 \cdot 8$ & 0.0 & $8 \cdot 8$ & $37 \cdot 0$ & $105 \cdot 2$ & $163 \cdot 0$ & $11 \cdot 2$ & 1.88 \\
\hline Berries & $14 \cdot 1$ & $28 \cdot 8$ & 0.3 & 1.6 & $6 \cdot 4$ & $14 \cdot 4$ & $34 \cdot 8$ & 9.7 & 1.63 \\
\hline Other fruits & $232 \cdot 6$ & 213.4 & $48 \cdot 0$ & $101 \cdot 7$ & $181 \cdot 8$ & 294.8 & $457 \cdot 6$ & 0.6 & 2.72 \\
\hline Dried fruits & $10 \cdot 6$ & $20 \cdot 9$ & 0.0 & 0.7 & $3 \cdot 3$ & $12 \cdot 5$ & $27 \cdot 1$ & $22 \cdot 5$ & 11.59 \\
\hline Tomatoes & $120 \cdot 1$ & $105 \cdot 0$ & $25 \cdot 2$ & $52 \cdot 6$ & 95.9 & $156 \cdot 0$ & $235 \cdot 6$ & 0.4 & $1 \cdot 17$ \\
\hline Leafy greens & 42.9 & $48 \cdot 0$ & $3 \cdot 8$ & $12 \cdot 1$ & $30 \cdot 0$ & $55 \cdot 9$ & $96 \cdot 2$ & $4 \cdot 1$ & 0.92 \\
\hline Cruciferous vegetables & $30 \cdot 2$ & $32 \cdot 7$ & $5 \cdot 7$ & $11 \cdot 4$ & $19 \cdot 5$ & $39 \cdot 1$ & $68 \cdot 1$ & $2 \cdot 8$ & $1 \cdot 26$ \\
\hline Onions & $26 \cdot 6$ & $29 \cdot 7$ & $2 \cdot 4$ & $6 \cdot 3$ & $17 \cdot 8$ & $39 \cdot 8$ & $60 \cdot 6$ & 1.4 & 1.80 \\
\hline Other vegetables & $107 \cdot 4$ & $110 \cdot 2$ & 24.5 & $44 \cdot 0$ & $78 \cdot 6$ & $133 \cdot 7$ & $214 \cdot 1$ & 0.1 & 1.88 \\
\hline Avocados & $7 \cdot 0$ & $14 \cdot 1$ & 0.0 & 0.0 & $4 \cdot 3$ & $6 \cdot 4$ & $27 \cdot 4$ & $42 \cdot 6$ & $5 \cdot 19$ \\
\hline Sweet potatoes & $5 \cdot 2$ & $9 \cdot 1$ & 0.0 & 0.0 & $4 \cdot 0$ & $6 \cdot 0$ & $8 \cdot 6$ & 34.5 & $3 \cdot 77$ \\
\hline White potatoes & 22.9 & $23 \cdot 0$ & $5 \cdot 0$ & $7 \cdot 4$ & $14 \cdot 8$ & $36 \cdot 2$ & $52 \cdot 5$ & $5 \cdot 8$ & $3 \cdot 72$ \\
\hline Fried potatoes & $10 \cdot 1$ & $17 \cdot 4$ & 0.0 & 0.0 & $6 \cdot 6$ & $10 \cdot 0$ & $21 \cdot 2$ & $38 \cdot 3$ & $10 \cdot 17$ \\
\hline Whole grains & $187 \cdot 3$ & $150 \cdot 1$ & 35.4 & 73.9 & $148 \cdot 8$ & $262 \cdot 8$ & $385 \cdot 2$ & 0.9 & 7.53 \\
\hline Refined grains & $105 \cdot 9$ & $95 \cdot 0$ & 21.9 & $42 \cdot 1$ & $79 \cdot 0$ & $139 \cdot 6$ & $220 \cdot 7$ & 0.6 & $7 \cdot 11$ \\
\hline Mixed grains & $3 \cdot 2$ & $14 \cdot 2$ & 0.0 & 0.0 & 0.0 & 0.0 & $7 \cdot 5$ & $76 \cdot 7$ & $12 \cdot 43$ \\
\hline Legumes & $62 \cdot 0$ & $63 \cdot 0$ & $10 \cdot 2$ & $23 \cdot 2$ & $43 \cdot 3$ & 80.9 & $134 \cdot 5$ & 3.9 & 4.98 \\
\hline Meat analogues & $45 \cdot 2$ & $54 \cdot 3$ & 0.0 & $9 \cdot 3$ & $31 \cdot 3$ & $61 \cdot 6$ & $103 \cdot 9$ & $16 \cdot 2$ & $7 \cdot 53$ \\
\hline Soyabeans and tofu & $16 \cdot 5$ & 33.5 & $0 \cdot 0$ & 0.0 & $6 \cdot 0$ & $12 \cdot 8$ & $57 \cdot 7$ & $41 \cdot 4$ & $5 \cdot 77$ \\
\hline Soya milk & 63.6 & $139 \cdot 5$ & 0.0 & 0.0 & 0.0 & $70 \cdot 1$ & $236 \cdot 7$ & $59 \cdot 2$ & 1.84 \\
\hline Peanuts & 2.5 & $6 \cdot 0$ & 0.0 & 0.0 & $1 \cdot 2$ & $1 \cdot 8$ & $7 \cdot 7$ & $38 \cdot 2$ & 25.02 \\
\hline Peanut butter & $5 \cdot 3$ & $7 \cdot 8$ & 0.0 & $1 \cdot 1$ & $2 \cdot 3$ & $6 \cdot 9$ & $12 \cdot 7$ & $19 \cdot 2$ & 24.60 \\
\hline Tree nuts & $10 \cdot 0$ & $15 \cdot 6$ & 0.0 & 1.4 & $4 \cdot 1$ & $13 \cdot 3$ & 25.5 & 13.9 & $25 \cdot 56$ \\
\hline Mixed nuts & $2 \cdot 8$ & 6.5 & 0.0 & 0.0 & $1 \cdot 3$ & $2 \cdot 0$ & $8 \cdot 4$ & $41 \cdot 0$ & 24.85 \\
\hline Seeds & $3 \cdot 0$ & $7 \cdot 7$ & 0.0 & 0.0 & 0.0 & $2 \cdot 2$ & $9 \cdot 3$ & $52 \cdot 3$ & $23 \cdot 81$ \\
\hline Unprocessed red meat & $7 \cdot 2$ & $17 \cdot 1$ & 0.0 & 0.0 & 0.0 & $8 \cdot 0$ & $20 \cdot 7$ & $64 \cdot 0$ & $10 \cdot 96$ \\
\hline Processed red meat & 0.7 & $2 \cdot 8$ & 0.0 & 0.0 & 0.0 & 0.0 & 1.4 & $84 \cdot 2$ & 12.43 \\
\hline Unprocessed poultry & 9.5 & $18 \cdot 4$ & 0.0 & 0.0 & 0.0 & $11 \cdot 0$ & $33 \cdot 1$ & $51 \cdot 6$ & 7.95 \\
\hline Processed poultry & 0.6 & $1 \cdot 7$ & 0.0 & 0.0 & 0.0 & 0.6 & 1.4 & 71.5 & 4.52 \\
\hline Fatty fish & 3.5 & $8 \cdot 3$ & 0.0 & 0.0 & 0.0 & $5 \cdot 7$ & $8 \cdot 6$ & 64.9 & $7 \cdot 61$ \\
\hline Other fish & 6.5 & $12 \cdot 9$ & 0.0 & 0.0 & 0.0 & 9.5 & $16 \cdot 0$ & $51 \cdot 2$ & 5.44 \\
\hline Regular milk products & $57 \cdot 9$ & $131 \cdot 7$ & 0.0 & 0.0 & $9 \cdot 0$ & $45 \cdot 4$ & $191 \cdot 8$ & $26 \cdot 2$ & $2 \cdot 72$ \\
\hline $\begin{array}{l}\text { Reduced-fat } \\
\text { milk products }\end{array}$ & $67 \cdot 6$ & $152 \cdot 1$ & 0.0 & 0.0 & $6 \cdot 7$ & $80 \cdot 8$ & 205.5 & $49 \cdot 0$ & $1 \cdot 88$ \\
\hline Cheese & $20 \cdot 8$ & $27 \cdot 0$ & 0.0 & 3.9 & $12 \cdot 4$ & $26 \cdot 5$ & 53.4 & $13 \cdot 2$ & $10 \cdot 08$ \\
\hline Eggs & 9.6 & $15 \cdot 9$ & 0.0 & 0.0 & $4 \cdot 1$ & $17 \cdot 5$ & $26 \cdot 2$ & $26 \cdot 1$ & $8 \cdot 41$ \\
\hline Butter & $2 \cdot 7$ & $6 \cdot 9$ & 0.0 & 0.0 & 0.2 & 1.9 & $8 \cdot 3$ & $29 \cdot 4$ & 29.96 \\
\hline Solid fats & $9 \cdot 3$ & $13 \cdot 3$ & 0.0 & 0.9 & $5 \cdot 2$ & $12 \cdot 9$ & $22 \cdot 3$ & $19 \cdot 0$ & $29 \cdot 37$ \\
\hline Salad dressings & $18 \cdot 1$ & $17 \cdot 7$ & 1.6 & $5 \cdot 2$ & $13 \cdot 2$ & $26 \cdot 7$ & $40 \cdot 8$ & $8 \cdot 0$ & $13 \cdot 72$ \\
\hline Liquid fats & $10 \cdot 7$ & $12 \cdot 6$ & $1 \cdot 1$ & 2.9 & $6 \cdot 6$ & $14 \cdot 1$ & $24 \cdot 7$ & 1.4 & 36.99 \\
\hline Coconut milk & 0.5 & 3.9 & 0.0 & 0.0 & 0.0 & 0.0 & 0.0 & $92 \cdot 5$ & 8.45 \\
\hline Dairy desserts & $19 \cdot 4$ & $37 \cdot 4$ & 0.0 & 0.0 & $10 \cdot 6$ & $18 \cdot 5$ & $67 \cdot 7$ & $35 \cdot 1$ & $5 \cdot 23$ \\
\hline Other desserts & $20 \cdot 1$ & 30.9 & 0.0 & 3.6 & $12 \cdot 1$ & $22 \cdot 4$ & $49 \cdot 6$ & $8 \cdot 8$ & $16 \cdot 69$ \\
\hline Snack foods & 3.6 & 5.9 & 0.0 & 0.6 & $2 \cdot 0$ & $4 \cdot 3$ & $9 \cdot 1$ & $19 \cdot 5$ & $26 \cdot 69$ \\
\hline Coffee & $73 \cdot 3$ & 183.9 & 0.0 & 0.0 & 0.0 & $25 \cdot 6$ & $253 \cdot 0$ & $66 \cdot 2$ & 0.08 \\
\hline Tea & $34 \cdot 3$ & $109 \cdot 0$ & 0.0 & 0.0 & 0.0 & 11.9 & $76 \cdot 2$ & 63.4 & 0.04 \\
\hline Soda & $132 \cdot 7$ & $329 \cdot 3$ & 0.0 & 0.0 & $23 \cdot 8$ & $127 \cdot 8$ & $372 \cdot 0$ & $47 \cdot 4$ & 0.79 \\
\hline
\end{tabular}


Table 1. Continued

\begin{tabular}{|c|c|c|c|c|c|c|c|c|c|}
\hline Foods & $\begin{array}{l}\text { Mean } \\
(\mathrm{g})\end{array}$ & $\begin{array}{l}\text { SD } \\
(g)\end{array}$ & $\begin{array}{l}\text { 10th percentile } \\
\text { (g) }\end{array}$ & $\begin{array}{l}\text { 25th } \\
\text { percentile } \\
\text { (g) }\end{array}$ & $\begin{array}{l}\text { Median } \\
\text { (g) }\end{array}$ & $\begin{array}{c}\text { 75th } \\
\text { percentile } \\
\text { (g) }\end{array}$ & $\begin{array}{c}\text { 90th } \\
\text { percentile } \\
\text { (g) }\end{array}$ & $\begin{array}{c}\text { Zero } \\
\text { intake† (\%) }\end{array}$ & $\begin{array}{l}\text { Density } \ddagger \\
(\mathrm{kJ} / \mathrm{g})\end{array}$ \\
\hline Fruit juices & 139.5 & $202 \cdot 2$ & 0.0 & $16 \cdot 7$ & $71 \cdot 0$ & $212 \cdot 8$ & $318 \cdot 3$ & $15 \cdot 4$ & 1.88 \\
\hline $\begin{array}{l}\text { Meal replacement } \\
\text { drinks }\end{array}$ & $18 \cdot 0$ & 78.8 & 0.0 & 0.0 & 0.0 & 0.0 & $18 \cdot 1$ & $85 \cdot 2$ & 3.35 \\
\hline Alcoholic beverages & 4.5 & $47 \cdot 3$ & 0.0 & 0.0 & 0.0 & 0.0 & 0.0 & 95.4 & 2.59 \\
\hline Hot cocoa & $10 \cdot 9$ & 44.5 & 0.0 & 0.0 & 0.0 & 0.0 & $20 \cdot 1$ & $76 \cdot 4$ & $2 \cdot 80$ \\
\hline Non-dairy milk & 6.5 & $40 \cdot 0$ & 0.0 & 0.0 & 0.0 & 0.0 & 0.0 & 94.5 & 2.09 \\
\hline Drinking-water & $1122 \cdot 9$ & 594.6 & 236.9 & $592 \cdot 3$ & $1066 \cdot 1$ & 1539.9 & $1599 \cdot 1$ & $2 \cdot 3$ & 0.00 \\
\hline
\end{tabular}

* Unadjusted values. Not standardised to an $8368 \mathrm{~kJ} / \mathrm{d}(2000 \mathrm{kcal} / \mathrm{d})$ diet.

$\dagger$ Percentages of participants who reported zero intake of the food.

$\ddagger$ Energy density of the food group.

\section{Food consumption by dietary pattern: minor food groups}

As with the major food groups, the four vegetarian dietary patterns differed significantly from the non-vegetarian dietary pattern with respect to the consumption of all minor food groups examined. The relative mean daily consumption of each minor food group for the four vegetarian dietary patterns, compared with the non-vegetarian dietary pattern (assigned the reference value of 1.00 for each food group), adjusted for age, sex and race and standardised to an $8368 \mathrm{~kJ} / \mathrm{d}(2000 \mathrm{kcal} / \mathrm{d})$ diet, is summarised in Table 3 . The absolute amounts consumed are given in Table 2 .

Vegetarians, particularly vegans, consumed moderately more citrus fruits, berries and other fresh fruits when compared with non-vegetarians; the differences were even greater for dried fruits. Vegetarians consumed only modestly more tomatoes. Vegans and pesco-vegetarians consumed higher amounts of leafy greens, cruciferous vegetables, onions and other vegetables, whereas lacto-ovo-vegetarians and semi-vegetarians consumed amounts similar to those consumed by non-vegetarians. Vegetarians, particularly vegans, consumed considerably more avocados when compared with non-vegetarians.

The situation was more nuanced in the case of starchy foods. Vegetarians consumed lower amounts of fried potatoes when compared with non-vegetarians. Vegans and pesco-vegetarians consumed more sweet potatoes when compared with non-vegetarians, whereas lacto-ovo-vegetarians and semivegetarians consumed slightly less. Vegetarians, except pescovegetarians, consumed more (non-fried) white potatoes when compared with non-vegetarians. Vegetarians consumed more whole grains and mixed grains when compared with nonvegetarians; however, vegetarians consumed less refined grains.

In the case of plant protein food groups, vegetarians were found to consume considerably more legumes, meat analogues, soyabeans and tofu, and soya milk when compared with non-vegetarians. Vegetarians consumed moderately more peanut butter, but similar amounts of peanuts. Vegetarians consumed more tree nuts and seeds when compared with non-vegetarians, with vegans consuming notably increased amounts.

Semi-vegetarians consumed considerably less processed red meat, unprocessed red meat, processed poultry, unprocessed poultry, fatty fish and other fish when compared with nonvegetarians. Pesco-vegetarians consumed fatty fish and other fish in amounts similar to those consumed by non-vegetarians and much higher than those consumed by semi-vegetarians. The consumption of meats, particularly processed meats, was quite low even among non-vegetarians (Table 2). Vegans did not consume or consumed trivial amounts of eggs, cheeses, reduced-fat milks and regular milks, as expected; semi-vegetarians consumed only modestly less when compared with non-vegetarians, and pesco-vegetarians and lacto-ovo-vegetarians consumed moderately less when compared with non-vegetarians.

Although vegetarians consumed lower amounts of added fats overall, differences emerged within this category. Vegetarians, particularly vegans, consumed reduced amounts of butter. Vegetarians also consumed notably lower amounts of solid fats (i.e. margarines and shortenings) when compared with non-vegetarians and lower amounts of salad dressings. On the other hand, vegans and pesco-vegetarians consumed more liquid fats (i.e. oils) when compared with non-vegetarians. The consumption of coconut milk was highest among pescovegetarians, followed by vegans and non-vegetarians, and lowest among lacto-ovo-vegetarians and semi-vegetarians. In the case of sweets, vegetarians (especially vegans) were found to consume considerably lower amounts of not only dairy desserts but also other desserts when compared with non-vegetarians. Vegetarians, particularly vegans and pescovegetarians, also consumed lower amounts of snack foods.

In the case of beverages, vegetarians (especially vegans) were found to drink dramatically less soda, coffee and alcohol when compared with non-vegetarians. Differences in the consumption of fruit juices were less striking, but vegans drank the least. Vegans, lacto-ovo-vegetarians and semivegetarians drank modestly less herbal tea when compared with non-vegetarians and pesco-vegetarians drank modestly more. The consumption of both meal replacement drinks and hot cocoa was dramatically lower among vegans than among the other dietary groups. The consumption of non-dairy milk (principally rice milk, as soya milk is categorised elsewhere) was highest in the vegan group, lowest in the non-vegetarian group and intermediate in the other dietary groups. Vegetarians also drank moderately higher quantities of water. 
Table 2. Daily mean consumption ( $\mathrm{g} / \mathrm{d})$ of food groups according to dietary pattern, minimally adjusted ${ }^{*} \dagger$

\begin{tabular}{|c|c|c|c|c|c|}
\hline Foods & Vegan & Lacto-ovo vegetarian & Pesco-vegetarian & Semi-vegetarian & Non-vegetariar \\
\hline \multicolumn{6}{|l|}{ Major food groups } \\
\hline Fruits & $483 \cdot 1$ & $357 \cdot 0$ & $400 \cdot 3$ & 343.0 & 298.8 \\
\hline Vegetables & $424 \cdot 1$ & $347 \cdot 2$ & $386 \cdot 0$ & 337.0 & 319.9 \\
\hline Avocados & 14.7 & 8.5 & 8.7 & $7 \cdot 0$ & $5 \cdot 3$ \\
\hline Potatoes & $42 \cdot 2$ & 40.9 & 36.5 & 39.9 & $40 \cdot 0$ \\
\hline Grains & 371.6 & $315 \cdot 6$ & 319.8 & $306 \cdot 6$ & $285 \cdot 3$ \\
\hline Legumes & 84.4 & 73.4 & $75 \cdot 2$ & 65.5 & 52.5 \\
\hline Soya foods and meat analogues & 202.9 & $166 \cdot 4$ & $172 \cdot 6$ & $136 \cdot 2$ & $88 \cdot 1$ \\
\hline Nuts and seeds & 36.0 & 27.5 & $25 \cdot 0$ & 23.4 & $18 \cdot 8$ \\
\hline Meat & 0.0 & 0.0 & $18 \cdot 2$ & $9 \cdot 1$ & $59 \cdot 2$ \\
\hline Dairy products & $2 \cdot 1$ & $120 \cdot 5$ & 114.4 & $177 \cdot 8$ & $200 \cdot 3$ \\
\hline Eggs & 0.0 & 7.6 & 8.3 & $10 \cdot 6$ & 14.1 \\
\hline Added fats & $25 \cdot 9$ & $41 \cdot 0$ & 40.6 & $43 \cdot 7$ & $46 \cdot 3$ \\
\hline Sweets & 8.6 & $37 \cdot 0$ & $32 \cdot 2$ & 42.5 & 49.5 \\
\hline Snack foods & 3.5 & 3.9 & 3.2 & $4 \cdot 1$ & 4.0 \\
\hline Beverages & $187 \cdot 8$ & $314 \cdot 2$ & $372 \cdot 8$ & 466.8 & $597 \cdot 3$ \\
\hline Drinking-water & $1629 \cdot 3$ & $1421 \cdot 3$ & $1304 \cdot 9$ & $1445 \cdot 8$ & 1191.0 \\
\hline \multicolumn{6}{|l|}{ Minor food groups } \\
\hline Citrus fruits & 108.5 & 81.5 & $86 \cdot 3$ & $76 \cdot 4$ & $63 \cdot 7$ \\
\hline Berries & 20.7 & $16 \cdot 1$ & $16 \cdot 8$ & 14.0 & 11.8 \\
\hline Other fruits & 334.4 & 247.0 & 284.8 & $242 \cdot 3$ & $215 \cdot 7$ \\
\hline Dried fruits & 19.5 & $12 \cdot 3$ & 12.4 & 10.4 & 7.6 \\
\hline Tomatoes & $137 \cdot 3$ & 134.8 & $130 \cdot 0$ & 129.9 & $117 \cdot 2$ \\
\hline Leafy greens & 58.9 & 44.9 & 54.5 & 43.6 & 42.5 \\
\hline Cruciferous vegetables & 44.5 & 31.4 & 36.7 & $30 \cdot 3$ & $29 \cdot 6$ \\
\hline Onions & 34.2 & $26 \cdot 3$ & 32.5 & $27 \cdot 2$ & $26 \cdot 9$ \\
\hline Other vegetables & $149 \cdot 1$ & 109.7 & $132 \cdot 4$ & $106 \cdot 1$ & 103.8 \\
\hline Avocados & 14.7 & 8.5 & 8.7 & $7 \cdot 0$ & $5 \cdot 3$ \\
\hline Sweet potatoes & 7.6 & $5 \cdot 0$ & 6.9 & 4.9 & $5 \cdot 2$ \\
\hline White potatoes & 28.9 & $27 \cdot 0$ & 21.9 & $24 \cdot 8$ & 21.9 \\
\hline Fried potatoes & $5 \cdot 8$ & 8.9 & 7.7 & $10 \cdot 2$ & 12.9 \\
\hline Whole grains & 292.8 & 214.0 & 210.6 & $197 \cdot 8$ & 157.9 \\
\hline Refined grains & 74.5 & 98.1 & $105 \cdot 7$ & $105 \cdot 2$ & 124.5 \\
\hline Mixed grains & 4.3 & 3.5 & 3.5 & 3.7 & 2.9 \\
\hline Legumes & 84.4 & 73.4 & $75 \cdot 2$ & 65.5 & 52.5 \\
\hline Meat analogues & 43.4 & $62 \cdot 1$ & 59.7 & $56 \cdot 4$ & $35 \cdot 6$ \\
\hline Soyabeans and tofu & $40 \cdot 2$ & $21 \cdot 6$ & 23.6 & 13.6 & 8.5 \\
\hline Soya milk & $119 \cdot 3$ & $82 \cdot 8$ & $89 \cdot 4$ & $66 \cdot 2$ & $44 \cdot 1$ \\
\hline Peanuts & $2 \cdot 1$ & $2 \cdot 4$ & $2 \cdot 7$ & $2 \cdot 4$ & $2 \cdot 6$ \\
\hline Peanut butter & 6.3 & 6.7 & 4.9 & 5.9 & 4.5 \\
\hline Tree nuts & 17.8 & $12 \cdot 1$ & $11 \cdot 0$ & $9 \cdot 6$ & $7 \cdot 1$ \\
\hline Mixed nuts & 2.5 & $2 \cdot 9$ & 3.2 & $2 \cdot 7$ & $2 \cdot 6$ \\
\hline Seeds & 7.3 & 3.4 & 3.1 & $2 \cdot 7$ & $2 \cdot 0$ \\
\hline Unprocessed red meat & 0.0 & 0.0 & 0.0 & 1.6 & $16 \cdot 1$ \\
\hline Processed red meat & 0.0 & 0.0 & 0.0 & 0.1 & 1.4 \\
\hline Unprocessed poultry & 0.0 & 0.0 & 0.0 & $5 \cdot 2$ & $21 \cdot 7$ \\
\hline Processed poultry & 0.0 & 0.0 & 0.0 & 0.2 & 1.3 \\
\hline Fatty fish & 0.0 & 0.0 & 7.5 & 0.8 & $6 \cdot 3$ \\
\hline Other fish & 0.0 & 0.0 & $10 \cdot 7$ & 1.3 & $12 \cdot 3$ \\
\hline Regular milk products & 0.7 & $38 \cdot 3$ & $40 \cdot 0$ & $65 \cdot 1$ & $84 \cdot 2$ \\
\hline Reduced-fat milk products & 0.0 & $60 \cdot 8$ & $56 \cdot 8$ & $87 \cdot 1$ & $90 \cdot 4$ \\
\hline Cheese & 1.4 & $21 \cdot 3$ & $17 \cdot 7$ & $25 \cdot 6$ & $25 \cdot 7$ \\
\hline Eggs & 0.0 & 7.6 & $8 \cdot 3$ & $10 \cdot 6$ & $14 \cdot 1$ \\
\hline Butter & 0.1 & 1.9 & $2 \cdot 3$ & 2.9 & 3.9 \\
\hline Solid fats & 3.9 & 9.5 & $7 \cdot 7$ & $10 \cdot 4$ & 10.5 \\
\hline Salad dressings & 8.5 & $18 \cdot 7$ & $16 \cdot 8$ & $19 \cdot 8$ & $21 \cdot 1$ \\
\hline Liquid fats & $12 \cdot 7$ & $10 \cdot 6$ & $12 \cdot 8$ & $10 \cdot 3$ & $10 \cdot 3$ \\
\hline Coconut milk & 0.7 & 0.3 & 1.0 & 0.3 & 0.6 \\
\hline Dairy desserts & 0.0 & $18 \cdot 1$ & $15 \cdot 9$ & $22 \cdot 1$ & 25.5 \\
\hline Other desserts & 8.6 & 18.9 & $16 \cdot 3$ & $20 \cdot 4$ & $24 \cdot 1$ \\
\hline Snack foods & 3.5 & 3.9 & 3.2 & $4 \cdot 1$ & $4 \cdot 0$ \\
\hline Coffee & 9.9 & $36 \cdot 1$ & 50.6 & 99.0 & 134.4 \\
\hline Tea & 37.2 & $25 \cdot 8$ & $56 \cdot 2$ & $30 \cdot 0$ & 41.8 \\
\hline Soda & $15 \cdot 3$ & $83 \cdot 1$ & $66 \cdot 0$ & $149 \cdot 1$ & 223.4 \\
\hline Fruit juices & $108 \cdot 4$ & $133 \cdot 3$ & $159 \cdot 4$ & $146 \cdot 8$ & 149.9 \\
\hline Meal replacement drinks & 0.0 & $16 \cdot 4$ & 17.4 & $21 \cdot 0$ & 22.4 \\
\hline Alcoholic beverages & 0.2 & 1.0 & $2 \cdot 3$ & 3.3 & 8.4 \\
\hline Hot cocoa & 0.0 & 9.4 & 11.8 & $10 \cdot 1$ & 13.5 \\
\hline Non-dairy milk & 16.9 & $9 \cdot 1$ & $9 \cdot 2$ & $7 \cdot 4$ & 3.4 \\
\hline Drinking-water & $1629 \cdot 3$ & $1421 \cdot 3$ & 1304.9 & $1445 \cdot 8$ & $1191 \cdot 0$ \\
\hline
\end{tabular}

* Adjusted for age (seven categories), sex and race (black $v$. non-black) by direct standardisation and standardised to an $8368 \mathrm{~kJ} / \mathrm{d}(2000 \mathrm{kcal} / \mathrm{d})$ diet. $\dagger P<0.0001$ for each food group. $P$ value obtained from a $\chi^{2}$ test with $4 \mathrm{df}$ testing the null hypothesis that the mean consumption of a food group for all the four vegetarian dietary patterns is the same as that for the non-vegetarian dietary pattern. 


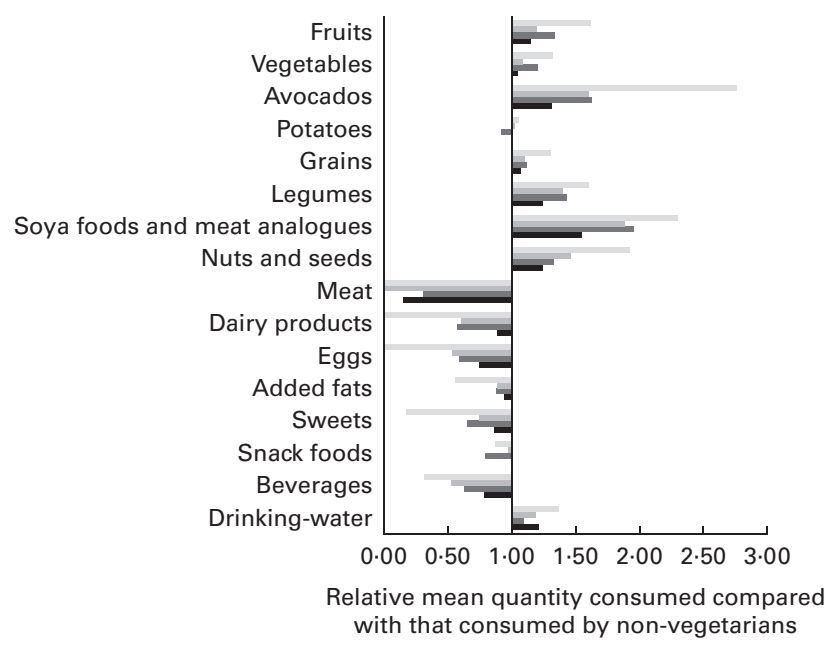

Fig. 1. Comparative consumption of major food groups by vegetarians and non-vegetarians. The relative mean (the ratio of the standardised mean in $\mathrm{g}$ for each vegetarian dietary pattern to the standardised mean in $\mathrm{g}$ for the non-vegetarian dietary pattern) quantity (in g) is shown for each major food group after adjustment for age (seven categories), sex and race (black $v$. non-black) by direct standardisation and after standardisation to an $8368 \mathrm{~kJ} / \mathrm{d}$ $(2000 \mathrm{kcal} / \mathrm{d})$ diet. $\square$, Vegan $v$. non-vegetarian; $\square$, lacto-ovo-vegetarian $v$. non-vegetarian; $\square$, pesco-vegetarian $v$. non-vegetarian; $\mathbf{c}$, semi-vegetarian v. non-vegetarian.

\section{Discussion}

The present study provides an important characterisation of how several vegetarian dietary patterns differ from a nonvegetarian dietary pattern as to the types of foods consumed. In a previous paper examining nutrient profiles, similar total food intakes by weight (in g) and similar energy intakes were found for all the dietary patterns ${ }^{(8)}$. Comparisons of food consumption patterns were also adjusted by standardising to an $8368 \mathrm{~kJ} / \mathrm{d}(2000 \mathrm{kcal} / \mathrm{d})$ diet to ensure that the reported differences in food group-specific consumption were independent of any differences in total energy intake (although these adjustments led to little substantive difference in the results). Thus, it is expected that vegetarians would consume higher amounts of certain foods of plant origin to make up for the lack of animal protein foods in their diet. However, it is difficult to predict the consumption of which plant foods might be increased, and it is likely that the health consequences of vegetarian diets might be contingent on this question. The present study provides helpful insight into this issue in a large group of North American vegetarians.

It is notable that moderate to large increases in the consumption of a broad spectrum of foods of plant origin including legumes, soya foods and meat analogues, nuts and seeds, grains, potatoes, avocados, fruits and vegetables, rather than a concentrated increase in that of only a few food groups, were observed for vegetarian dietary patterns. Such diversity would be expected to be helpful in terms of nutritional adequacy. This is consistent with the analysis of nutrient profiles of vegetarian dietary patterns carried out by Rizzo et $a{ }^{(8)}$. In addition, this increased consumption of many plant foods would be expected to result in higher intakes of a variety of phytochemicals, many of which are hypothesised to confer health benefits. Furthermore, evidence linking increased consumption of a number of these plant foods to health benefits exists. The consumption of nuts has been linked to reduced CVD risk and increased longevity ${ }^{(17-19)}$. Increased consumption of fruits and vegetables may be linked to a lower risk of certain cancers ${ }^{(20)}$.

It might be expected that lacto-ovo-vegetarians would have consumed higher amounts of dairy products and eggs compared with non-vegetarians to make up for the lack of meat in the diet. In fact, the consumption of these foods was reduced in this group. Pesco-vegetarians and semi-vegetarians also consumed reduced amounts of dairy products and eggs when compared with non-vegetarians. Thus, the consumption of dairy products and eggs tended to correlate with that of meat.

Perhaps more worth noting still are the foods consumed less by vegetarians in the present study apart from meats, eggs and dairy products - primarily added fats, sweets, snack foods, non-water beverages and refined grains. This is interesting both for its potential health impact and in terms of insight into the dietary decision-making of the vegetarians in this cohort.

Vegetarians consumed reduced amounts of butter and solid fats, but comparable or increased amounts of liquid fats (i.e. oils) when compared with non-vegetarians. This is consistent with dietary recommendations to replace solid fats with oils ${ }^{(21)}$, based on evidence that substituting unsaturated fatty acids for SFA reduces heart disease risk $^{(22,23)}$. Sweets and energy beverages such as soda and fruit juices are high in simple sugars in the form of sucrose, fructose and high-fructose maize syrup. Some evidence links increased consumption of sugars, and particularly fructose, to an increased risk of dyslipidaemia, insulin resistance, visceral adiposity and hepatic steatosis $^{(24-27)}$. Decreased consumption of these food groups might be responsible for some of the favourable associations previously demonstrated for vegetarians in this cohort including lower BMI, reduced prevalence of the metabolic syndrome and reduced incidence of diabetes mellitus type $\mathrm{II}^{(4,5)}$.

The food consumption patterns of vegetarians observed in the present study may provide some insight into their dietary decision-making. As has been mentioned previously, we defined the vegetarian dietary patterns on the basis of avoidance of certain foods of animal origin. We believe that this is consistent with common self-designations in the target population in the present study and the general public. For example, it is not unusual for people to self-designate as vegans or vegetarians. However, the present study demonstrates clear food consumption patterns among vegetarians that go well beyond avoidance of meats or other animal foods. Specifically, food consumption patterns among vegetarians are quite consistent with what is currently understood to constitute healthful food choices. The Dietary Guidelines for Americans in 2010, for example, emphasised increased consumption of fruits and vegetables, recommended decreased consumption of added sugars and solid fats, and favoured the consumption of whole grains over refined grains $^{(21)}$. The food consumption patterns of vegetarians in the present study, on average, are very consistent with such dietary guidelines. This appears to demonstrate that 
Table 3. Adjusted relative mean daily consumption of minor food groups for the four vegetarian dietary patterns compared with the non-vegetarian dietary pattern* $\dagger$

(Relative mean values and $95 \%$ confidence intervals $\ddagger$ )

\begin{tabular}{|c|c|c|c|c|c|c|c|c|}
\hline \multirow[b]{2}{*}{ Minor food groups } & \multicolumn{2}{|c|}{ Vegan } & \multicolumn{2}{|c|}{ Lacto-ovo vegetarian } & \multicolumn{2}{|c|}{ Pesco-vegetarian } & \multicolumn{2}{|c|}{ Semi-vegetarian } \\
\hline & $\begin{array}{c}\text { Relative } \\
\text { mean }\end{array}$ & $95 \% \mathrm{Cl}$ & Relative mean & $95 \% \mathrm{Cl}$ & Relative mean & $95 \% \mathrm{Cl}$ & Relative mean & $95 \% \mathrm{Cl}$ \\
\hline Citrus fruits & $1 \cdot 70$ & $1.65,1.75$ & $1 \cdot 28$ & $1.25,1.31$ & 1.35 & $1.31,1.40$ & $1 \cdot 20$ & $1 \cdot 15,1.25$ \\
\hline Berries & 1.75 & $1.67,1.82$ & 1.36 & $1.32,1.40$ & 1.42 & $1.35,1.49$ & $1 \cdot 18$ & $1 \cdot 11,1 \cdot 26$ \\
\hline Other fruits & 1.55 & $1.52,1.58$ & $1 \cdot 15$ & $1 \cdot 13,1 \cdot 16$ & 1.32 & $1 \cdot 30,1 \cdot 34$ & $1 \cdot 12$ & $1 \cdot 10,1 \cdot 15$ \\
\hline Dried fruits & 2.58 & $2 \cdot 48,2.67$ & 1.63 & $1.58,1.67$ & 1.64 & $1.57,1.71$ & 1.37 & $1.28,1.46$ \\
\hline Tomatoes & $1 \cdot 17$ & $1 \cdot 15,1 \cdot 20$ & $1 \cdot 15$ & $1 \cdot 14,1 \cdot 16$ & $1 \cdot 11$ & $1.09,1.13$ & $1 \cdot 11$ & $1.08,1.14$ \\
\hline Leafy greens & 1.39 & $1.35,1.42$ & 1.06 & $1.04,1.07$ & 1.28 & $1 \cdot 25,1 \cdot 31$ & 1.03 & $0.99,1.06$ \\
\hline Cruciferous vegetables & 1.51 & $1.47,1.55$ & 1.06 & $1.05,1.08$ & 1.24 & $1.21,1.27$ & 1.02 & $0.99,1.06$ \\
\hline Onions & $1 \cdot 27$ & $1.24,1.31$ & 0.98 & $0.96,1.00$ & 1.21 & $1 \cdot 18,1 \cdot 23$ & 1.01 & $0.98,1.04$ \\
\hline Other vegetables & 1.44 & $1.40,1.48$ & 1.06 & $1.04,1.07$ & 1.28 & $1 \cdot 24,1 \cdot 31$ & 1.02 & $0.99,1.05$ \\
\hline Avocados & 2.76 & $2.64,2.89$ & 1.60 & $1.55,1.65$ & 1.63 & $1.56,1.70$ & 1.31 & $1.23,1.39$ \\
\hline Sweet potatoes & 1.45 & $1.39,1.51$ & 0.96 & $0.94,0.98$ & 1.32 & $1.27,1.37$ & 0.94 & $0.89,0.99$ \\
\hline White potatoes & 1.32 & $1.28,1.35$ & 1.23 & $1.22,1.25$ & 1.00 & $0.98,1.02$ & $1 \cdot 13$ & $1 \cdot 10,1 \cdot 17$ \\
\hline Fried potatoes & 0.45 & $0.42,0.47$ & 0.69 & $0.67,0.71$ & 0.60 & $0.57,0.63$ & 0.79 & $0.75,0.84$ \\
\hline Whole grains & 1.85 & $1.83,1.88$ & 1.36 & $1.34,1.37$ & 1.33 & $1.31,1.36$ & 1.25 & $1 \cdot 22,1 \cdot 28$ \\
\hline Refined grains & 0.60 & $0.58,0.61$ & 0.79 & $0.78,0.80$ & 0.85 & $0.83,0.87$ & 0.84 & $0.82,0.87$ \\
\hline Mixed grains & 1.46 & $1.29,1.63$ & 1.21 & $1.13,1.29$ & 1.21 & $1.09,1.33$ & 1.25 & $1.10,1.39$ \\
\hline Legumes & 1.61 & $1.57,1.64$ & 1.40 & $1.38,1.42$ & 1.43 & $1.40,1.46$ & 1.25 & $1 \cdot 21,1.28$ \\
\hline Meat analogues & $1 \cdot 22$ & $1 \cdot 18,1.26$ & 1.75 & $1.72,1.77$ & 1.68 & $1.64,1.72$ & 1.58 & $1.54,1.63$ \\
\hline Soyabeans and tofu & 4.75 & $4.57,4.93$ & 2.55 & $2 \cdot 46,2.63$ & $2 \cdot 78$ & $2 \cdot 66,2.90$ & 1.60 & $1.50,1.70$ \\
\hline Soya milk & $2 \cdot 71$ & $2 \cdot 58,2.83$ & 1.88 & $1.81,1.94$ & 2.03 & $1.93,2.12$ & 1.50 & $1.40,1.61$ \\
\hline Peanuts & 0.80 & $0.75,0.85$ & 0.92 & $0.89,0.95$ & 1.07 & $1.02,1 \cdot 12$ & 0.94 & $0.87,1.01$ \\
\hline Peanut butter & 1.39 & $1.34,1.44$ & 1.49 & $1.46,1.52$ & 1.07 & $1.04,1.11$ & 1.31 & $1.25,1.36$ \\
\hline Tree nuts & 2.52 & $2.45,2.59$ & 1.71 & $1.68,1.75$ & 1.56 & $1.51,1.61$ & 1.36 & $1.30,1.41$ \\
\hline Mixed nuts & 0.97 & $0.90,1.03$ & $1 \cdot 10$ & $1.06,1.13$ & 1.21 & $1 \cdot 16,1 \cdot 27$ & 1.04 & $0.97,1.11$ \\
\hline Seeds & 3.73 & $3.55,3.91$ & 1.74 & $1.67,1.81$ & 1.59 & $1.50,1.69$ & 1.38 & $1.26,1.50$ \\
\hline Unprocessed red meat & \multicolumn{2}{|c|}{$0.00 \S$} & \multicolumn{2}{|c|}{$0.00 \S$} & \multicolumn{2}{|c|}{$0.00 \S$} & 0.10 & $0.09,0.11$ \\
\hline Processed red meat & \multicolumn{2}{|c|}{$0.00 \S$} & \multicolumn{2}{|c|}{$0.00 \S$} & \multicolumn{2}{|c|}{$0.00 \S$} & 0.05 & $0.04,0.06$ \\
\hline Unprocessed poultry & \multicolumn{2}{|c|}{$0.00 \S$} & \multicolumn{2}{|c|}{$0.00 \S$} & \multicolumn{2}{|c|}{$0.00 \S$} & 0.24 & $0.23,0.25$ \\
\hline Processed poultry & \multirow{2}{*}{\multicolumn{2}{|c|}{$\begin{array}{l}0.00 \S \\
0.00 \S\end{array}$}} & \multirow{2}{*}{\multicolumn{2}{|c|}{$0.00 \S$}} & \multicolumn{2}{|c|}{$0.00 \S$} & 0.12 & $0.11,0.13$ \\
\hline Fatty fish & & & 0.0 & & $1 \cdot 19$ & $1 \cdot 14,1 \cdot 23$ & 0.13 & $0.11,0.14$ \\
\hline Other fish & \multicolumn{2}{|c|}{$0.00 \S$} & 0.0 & & 0.87 & $0.84,0.90$ & 0.10 & $0.09,0.11$ \\
\hline Regular milk products & 0.01 & $0.01,0.01$ & 0.45 & $0.44,0.47$ & 0.47 & $0.45,0.50$ & 0.77 & $0.72,0.82$ \\
\hline Reduced-fat milk products & & & 0.67 & $0.65,0.70$ & 0.63 & $0.59,0.66$ & 0.96 & $0.90,1.03$ \\
\hline Cheese & 0.05 & $0.05,0.06$ & 0.83 & $0.81,0.85$ & 0.69 & $0.67,0.71$ & 1.00 & $0.96,1.03$ \\
\hline Eggs & & & 0.54 & $0.52,0.55$ & 0.59 & $0.57,0.61$ & 0.75 & $0.72,0.78$ \\
\hline Butter & 0.02 & $0.01,0.02$ & 0.49 & $0.47,0.51$ & 0.59 & $0.56,0.63$ & 0.75 & $0.69,0.80$ \\
\hline Solid fats & 0.38 & $0.36,0.40$ & 0.91 & $0.89,0.93$ & 0.73 & $0.71,0.76$ & 0.99 & $0.96,1.03$ \\
\hline Salad dressings & 0.40 & $0.39,0.42$ & 0.89 & $0.87,0.90$ & 0.80 & $0.78,0.81$ & 0.94 & $0.91,0.96$ \\
\hline Liquid fats & 1.22 & $1 \cdot 19,1 \cdot 26$ & 1.02 & $1.01,1.04$ & $1 \cdot 24$ & $1 \cdot 21,1 \cdot 27$ & 1.00 & $0.96,1.03$ \\
\hline Coconut milk & $1 \cdot 18$ & $0.99,1.37$ & 0.55 & $0.48,0.62$ & 1.78 & $1.55,2.00$ & 0.62 & $0.45,0.80$ \\
\hline Dairy desserts & & & 0.71 & $0.69,0.73$ & 0.62 & $0.59,0.65$ & 0.87 & $0.82,0.91$ \\
\hline Other desserts & 0.36 & $0.34,0.37$ & 0.79 & $0.77,0.80$ & 0.68 & $0.66,0.70$ & 0.85 & $0.81,0.89$ \\
\hline Snack foods & 0.87 & $0.84,0.91$ & 0.97 & $0.95,0.99$ & 0.79 & $0.76,0.83$ & 1.01 & $0.96,1.05$ \\
\hline Coffee & 0.07 & $0.05,0.09$ & 0.27 & $0.25,0.28$ & 0.38 & $0.35,0.41$ & 0.74 & $0.67,0.80$ \\
\hline Tea & 0.89 & $0.80,0.98$ & 0.62 & $0.58,0.66$ & 1.35 & $1.25,1.44$ & 0.72 & $0.64,0.80$ \\
\hline Soda & 0.07 & $0.05,0.08$ & 0.37 & $0.36,0.39$ & 0.30 & $0.27,0.32$ & 0.67 & $0.61,0.72$ \\
\hline Fruit juices & 0.72 & $0.69,0.75$ & 0.89 & $0.87,0.91$ & 1.06 & $1.03,1.10$ & 0.98 & $0.94,1.02$ \\
\hline Meal replacement drinks & & & 0.73 & $0.67,0.79$ & 0.77 & $0.69,0.86$ & 0.94 & $0.82,1.06$ \\
\hline Alcoholic beverages & 0.02 & $0.00,0.06$ & 0.12 & $0.09,0.15$ & 0.27 & $0.19,0.35$ & 0.40 & $0.19,0.60$ \\
\hline Hot cocoa & & & 0.69 & $0.65,0.74$ & 0.87 & $0.80,0.94$ & 0.74 & $0.65,0.83$ \\
\hline Non-dairy milk & 4.95 & $4 \cdot 32,5 \cdot 58$ & 2.66 & $2.39,2.94$ & 2.69 & $2 \cdot 32,3.07$ & $2 \cdot 18$ & $1 \cdot 76,2.59$ \\
\hline Drinking-water & 1.37 & $1.35,1.39$ & $1 \cdot 19$ & $1 \cdot 18,1 \cdot 21$ & $1 \cdot 10$ & $1.08,1.11$ & 1.21 & $1 \cdot 19,1.24$ \\
\hline
\end{tabular}

* The relative mean (the ratio of the standardised mean in $\mathrm{g}$ for each vegetarian dietary pattern to the standardised mean in $\mathrm{g}$ for the non-vegetarian dietary pattern) is shown for each food group after adjustment for age (seven categories), sex and race (black $v$. non-black) by direct standardisation and after standardisation to an $8368 \mathrm{~kJ} / \mathrm{d}$ $(2000 \mathrm{kcal} / \mathrm{d})$ diet.

$\dagger P<0.0001$ for each food group. $P$ value obtained from a $\chi^{2}$ test with $4 \mathrm{df}$ testing the null hypothesis that the mean consumption of a food group for all the four vegetarian dietary patterns is the same as that for the non-vegetarian dietary pattern.

$¥$ The $95 \% \mathrm{Cl}$ for the ratio of two means was calculated using a joint standard error estimate for the ratio of the two means, according to the following formula (a simplification of Fieller's theorem): $\mathrm{SE}_{Q}=Q \times\left(\mathrm{SE}_{A}^{2} / A^{2}+\mathrm{SE}_{B}^{2} / B^{2}\right)^{0.5}$, where $Q$ is the ratio of the means, $A$ is the first mean and $B$ is the second mean (i.e. non-vegetarian).

$\S \mathrm{Cl}$ not defined due to the zero intake of this food group in the specified vegetarian dietary pattern.

individuals in the present study choosing a vegetarian diet would also consciously make other healthful dietary choices. It is consistent with a health motivation for choosing a vegetarian diet.
These patterns may not be generalisable to all vegetarians. People elect vegetarian diets for a variety of reasons, including the desire for better health, ethical concerns, environmental considerations and religious beliefs. These underlying 
motivations may influence the choice of foods consumed, beyond the avoidance of meats and other animal foods. Although we do not have data to establish it, it is our belief that a desire for improved health and a belief that vegetarian diets are more healthful, partly informed by religious understandings, are major motivators for many Seventh-day Adventists to choose vegetarian diets. This health/religious motivation may also lead to the increased consumption of healthful plant food groups and the decreased consumption of added fats, sweets, snack foods, energy beverages and refined grains. In other vegetarian populations where motivations may differ, food choices may differ as well. For example, a vegetarian whose primary motivation is the avoidance of animal suffering may not necessarily drink less soda when compared with a non-vegetarian. Such differences could lead to some heterogeneous results among studies on the health effects of vegetarian diets.

The European Prospective Investigation into Cancer and Nutrition (EPIC)-Oxford cohort (also referred to as the EPIC British 'health-conscious' cohort) study is an important study of British vegetarians. There have been some differences in the results of studies on the association of vegetarian diets with certain health outcomes in the EPIC-Oxford study and the Adventist Health Studies, particularly for all-cause mortality and colon cancer, for which vegetarians in the Adventist Health Studies exhibited reduced risk, but vegetarians in EPIC-Oxford did not ${ }^{(7,28)}$. Observed differences in food consumption patterns between the two populations could be important to identify, as they might suggest possible explanations for these differing results. It has been noted that the intakes of vitamin $\mathrm{C}$ and dietary fibre are substantially higher among AHS-2 vegans than among EPIC-Oxford vegans ${ }^{(7)}$. Mean unadjusted consumption (in gram weight) for fruits and vegetables has been reported for a random sample of EPIC-Oxford cohort members as follows: $220 \cdot 9 \mathrm{~g}$, all vegetables; $18.4 \mathrm{~g}$, leafy green vegetables; $36.2 \mathrm{~g}$, cabbages; $13.4 \mathrm{~g}$, onions and garlic; $261.0 \mathrm{~g}$, all fruits; $57.0 \mathrm{~g}$, citrus fruits $^{(29)}$. Similar unadjusted means for the AHS-2 cohort (see Table 1) were as follows: $327 \cdot 1 \mathrm{~g}$ ( $48 \%$ higher), all vegetables; $42.9 \mathrm{~g}$ (133\% higher), leafy green vegetables (non-cruciferous); $30.2 \mathrm{~g}$ (17\% lower), cruciferous vegetables; $26.6 \mathrm{~g}$ (99\% higher), onions; 330.2 g (27\% higher), all fruits; $73.0 \mathrm{~g}$ ( $28 \%$ higher), citrus fruits. Although this is not a comparison of vegetarians from the two cohorts specifically, both cohorts have about half vegetarians and half non-vegetarians, and the AHS-2 cohort members overall clearly exhibit a considerably higher intake of most fruits and vegetables when compared with members of the EPIC-Oxford cohort.

These differences in health outcomes and in food consumption patterns may shed light on the types of vegetarian diets that are likely to be optimal. Although vegetarian diets have meat avoidance in common, they may differ in what takes meat's place in the diet. The AHS-2 vegetarian dietary patterns described herein may provide good examples of healthy approaches to replacing meat in the diet, primarily by increased consumption of a variety of whole plant foods. Given their association with reduced chronic disease risk and increased longevity, they probably provide a helpful illustration of what constitutes healthy plant-based dietary approaches. Future dietary guidelines might use this information to formulate recommendations.

In conclusion, we found that in the AHS-2 cohort, vegetarian dietary patterns are associated not only with reduced consumption of meats, eggs and dairy products, but also with increased consumption of a variety of plant foods and reduced consumption of added fats, sweets, snack foods, non-water beverages and refined grains. Vegetarian dietary patterns similar to those demonstrated in the present study population represent important, real-world dietary options with multiple simultaneous features that might be expected to confer health benefits such as protection against obesity and certain cardiometabolic diseases. Furthermore, these vegetarian dietary patterns have previously been reported to be associated with such beneficial outcomes. They may play an important role as models for dietetic counselling about healthy vegetarian diets and for future nutritional guidelines.

\section{Supplementary material}

To view supplementary material for this article, please visit http://dx.doi.org/10.1017/S000711451400261X

\section{Acknowledgements}

The authors thank Hanni Bennett, Research Associate, Adventist Health Studies, School of Public Health, Loma Linda University, for providing support to carry out the study.

The present study was supported by National Cancer Institute grant 1U01CA152939. The funding agency had no role in the design and analysis of the study or in the writing of this article. The views expressed in this article are those of the authors and do not necessarily represent the views of the National Cancer Institute. The ideas and opinions expressed herein are those of the authors and endorsement by the National Cancer Institute or its contractors or subcontractors is not intended and nor should it be inferred.

The authors' contributions are as follows: M. J. O., K. J.-S., J. S., P. N. S. and G. E. F. designed the study; M. J. O., K. J.-S. and J. F. conducted the study; M. J. O. analysed the data; M. J. O. wrote the article; G. E. F. had primary responsibility for the final content. All authors read and approved the final manuscript.

The authors report the following potential conflicts of interest: K. J.-S. has received research grants from the Almond Board of California and from Unilever. J. S. is an unpaid member of the Scientific Advisory Board for the California Walnut Commission and has received grants from the nut, soya and avocado growers.

The authors M. J. O., J. F., P. N. S. and G. E. F. have no conflicts of interest to declare.

\section{References}

1. Hu FB (2002) Dietary pattern analysis: a new direction in nutritional epidemiology. Curr Opin Lipidol 13, 3-9.

2. Fraser GE (2003) A search for truth in dietary epidemiology. Am J Clin Nutr 78, 521S-525S. 
3. Tonstad S, Butler TL, Yan R, et al. (2009) Type of vegetarian diet, body weight, and prevalence of type 2 diabetes. Diabetes Care 32, 791-796.

4. Rizzo NS, Sabate J, Jaceldo-Siegl K, et al. (2011) Vegetarian dietary patterns are associated with a lower risk of metabolic syndrome: The Adventist Health Study 2. Diabetes Care 34 , $1225-1227$.

5. Tonstad S, Stewart K, Oda K, et al. (2013) Vegetarian diets and incidence of diabetes in the Adventist Health Study-2. Nutr Metab Cardiovasc Dis 23, 292-299.

6. Pettersen BJ, Anousheh R, Fan J, et al. (2012) Vegetarian diets and blood pressure among white subjects: results from the Adventist Health Study-2 (AHS-2). Public Health Nutr 15, 1909-1916.

7. Orlich MJ, Singh PN, Sabate J, et al. (2013) Vegetarian dietary patterns and mortality in Adventist Health Study 2. JAMA Intern Med 173, 1230-1238.

8. Rizzo NS, Jaceldo-Siegl K, Sabate J, et al. (2013) Nutrient profiles of vegetarian and nonvegetarian dietary patterns. J Acad Nutr Diet 113, 1610-1619.

9. Butler TL, Fraser GE, Beeson WL, et al. (2008) Cohort profile: The Adventist Health Study-2 (AHS-2). Int J Epidemiol 37, $260-265$.

10. Jaceldo-Siegl K, Fan J, Sabate J, et al. (2011) Race-specific validation of food intake obtained from a comprehensive FFQ: the Adventist Health Study-2. Public Health Nutr 14, 1988-1997.

11. Jaceldo-Siegl K, Knutsen SF, Sabate J, et al. (2010) Validation of nutrient intake using an FFQ and repeated $24 \mathrm{~h}$ recalls in black and white subjects of the Adventist Health Study-2 (AHS-2). Public Health Nutr 13, 812-819.

12. Fieller EC (1940) The biological standardization of insulin. $J$ R Stat Soc Suppl. 7, 1-64.

13. Fraser GE \& Yan R (2007) Guided multiple imputation of missing data: using a subsample to strengthen the missingat-random assumption. Epidemiology 18, 246-252.

14. Fraser GE, Yan R, Butler TL, et al. (2009) Missing data in a long food frequency questionnaire: are imputed zeroes correct? Epidemiology 20, 289-294.

15. R Development Core Team (2012) R: A Language and Environment for Statistical Computing, 2nd ed. Vienna: R Foundation for Statistical Computing.
16. Harrell FE Jr, Dupont C, et al. (2014) Hmisc: Harrell Miscellaneous, 3rd ed. Nashville, TN. http://cran.r-project.org/ web/packages/Hmisc/Hmisc.pdf

17. Sabate J (1993) Does nut consumption protect against ischaemic heart disease? Eur J Clin Nutr 47, S71-S75.

18. Sabate J (1999) Nut consumption, vegetarian diets, ischemic heart disease risk, and all-cause mortality: evidence from epidemiologic studies. Am J Clin Nutr 70, 500S-503S.

19. Fraser GE \& Shavlik DJ (2001) Ten years of life: is it a matter of choice? Arch Intern Med 161, 1645-1652.

20. World Cancer Research Fund/American Institute for Cancer Research (2007) Food, Nutrition, Physical Activity, and the Prevention of Cancer: A Global Perspective. Washington, DC: AICR.

21. U.S. Department of Agriculture and U.S. Department of Health and Human Services (2010) Dietary Guidelines for Americans, 2010, 7th ed. Washington, DC: U.S. Government Printing Office.

22. Baum SJ, Kris-Etherton PM, Willett WC, et al. (2012) Fatty acids in cardiovascular health and disease: a comprehensive update. J Clin Lipidol 6, 216-234.

23. Willett WC (2012) Dietary fats and coronary heart disease. J Intern Med 272, 13-24.

24. Stanhope KL, Schwarz JM, Keim NL, et al. (2009) Consuming fructose-sweetened, not glucose-sweetened, beverages increases visceral adiposity and lipids and decreases insulin sensitivity in overweight/obese humans. J Clin Invest 119, $1322-1334$.

25. Vos MB \& Lavine JE (2013) Dietary fructose in nonalcoholic fatty liver disease. Hepatology 57, 2525-2531.

26. Tappy L \& Lê K-A (2010) Metabolic effects of fructose and the worldwide increase in obesity. Physiol Rev 90, 23-46.

27. Stanhope KL \& Havel PJ (2009) Fructose consumption: considerations for future research on its effects on adipose distribution, lipid metabolism, and insulin sensitivity in humans. J Nutr 139, 1236S-1241S.

28. Fraser GE (2009) Vegetarian diets: what do we know of their effects on common chronic diseases? Am J Clin Nutr 89 1607S-1612S.

29. Agudo A, Slimani N, Ocké MC, et al. (2002) Consumption of vegetables, fruit and other plant foods in the European Prospective Investigation into Cancer and Nutrition (EPIC) cohorts from 10 European countries. Public Health Nutr $\mathbf{5}$, 1179-1196. 